\title{
Effect of glass powder instead of mineral powder on asphalt mixture
}

\author{
Binkai Tang ${ }^{1, a^{*}}$, Hengshan Wu ${ }^{1, b}$, Yiping Liao ${ }^{1, c}$ and Dingping Huang ${ }^{2, d}$ \\ ${ }^{1}$ University of South China, Hengyang, Hunan,China \\ ${ }^{2}$ Hengyang Road Construction Co., Ltd, Hengyang, Hunan,China \\ a13928769372@163.com, b2638813275@qq.com, ${ }^{\circ} 121115819 @ q q . c o m,{ }^{d} 740005137 @ q q . c o m$
}

Keywords: Waste glass powder; Asphalt mixture;The optimum asphalt aggregate ratio; volume parameter; Water stability、

Abstract. This paper through the experiment discussed the glass powder to substitute the mineral powder to asphalt mixture effects of asphalt mixture aggregate ratio, volume parameters and water stability. Experimental results show that the glass powder to substitute the mineral powder have minimal impact to asphalt mixture, the volume parameter variation tendency of same in asphalt content by $4.4 \%$ gradually increased to $5.6 \%$, two kinds of asphalt mixture bulk relative density and asphalt saturation increases gradually and in bituminous dosage in 5.2\% achieved maximum value, after gradually reduced; two kinds of asphalt mixture voids in mineral aggregate gap rate decreased gradually and in $5.2 \%$ has a minimum value, then gradually increase. The stability of asphalt mixture doped glass powder is not decreased, at the same time the flow value is slightly larger than that of the asphalt mixture of the slag, but doped glass powder asphalt mixture water stability performance is inferior, except in extreme lack of rain conditions, not with glass powder doped added asphalt mixture.

\section{Introduction}

Glass asphalt concrete refers to the asphalt concrete in which the glass is replaced by a specific form of the asphalt concrete. At present, the technology of glass asphalt concrete has been more mature, and some parts of the United States have already worked out the corresponding operation criterion, and the New Jersey State Department of transportation in the United States put forward the mixed amount of glass on the basis of practical experience. It is not more than 10\%[3-5]. In China, Song Cheng [6] and other experimental studies show that, in order to ensure that the glass asphalt mixture has good structure, stability and good road performance, glass particle size is not greater than $5 \mathrm{~mm}$. In view of the reason why the water stability of the asphalt concrete is decreased, Hezheng studies show that the water stability of the asphalt concrete can be improved by the addition of anti scaling. But with glass powder instead of slag powder form, this research is not perfect. Therefore, in the form of the glass powder instead of mineral powder, glass powder and asphalt mixture volumetric parameter, water stable performance experiment, compared the performance changes. 


\section{Experime ntal materials and experimental content}

\section{Raw materials}

Glass powder: the is representative of the colorless glass beer bottles, clean up debris, dried, using a ball mill crushed to powder particle size.

The aggregate: the experimental use of basalt and limestone in Hengyang region. There are A, B, C, $\mathrm{D}$ four files aggregate and filler, where $\mathrm{A}$ material $(10 \sim 15 \mathrm{~mm})$ and $\mathrm{B}$ material $(5 \sim 10 \mathrm{~mm})$ are basalt, $\mathrm{C}$ aggregate $(3 \sim 5 \mathrm{~mm})$ and Daggre gate $(0 \sim 3 \mathrm{~mm})$ is limestone.

Asphalt: in view of the poor adhesion of glass and asphalt, the experimental use of the high melting point, good adhesion of the weight of 70A oil asphalt. Main performance index see table 1-1.

Table 1-1 :Asphalt index table

\begin{tabular}{ccccc}
\hline Asphalt type & $\begin{array}{c}\text { Penetration } \\
\left(25^{\circ} \mathrm{C}\right) /(0.1 \mathrm{~mm})\end{array}$ & $\begin{array}{c}\text { Softening point } \\
/{ }^{\circ} \mathrm{C}\end{array}$ & $\begin{array}{c}\text { Ductility } \\
\left(25^{\circ} \mathrm{C}\right) / \mathrm{cm}\end{array}$ & $\begin{array}{c}\text { Ductility } \\
\left(15^{\circ} \mathrm{C}\right) / \mathrm{cm}\end{array}$ \\
\hline $\begin{array}{c}\text { Heavy traffic asphalt } \\
70 \mathrm{~A}\end{array}$ & 66.9 & 47.9 & $>150$ & 55.1 \\
$\begin{array}{c}\text { Specification } \\
\text { requirements }\end{array}$ & $60 \sim 80$ & $\geq 46$ & $\geq 100$ & $\geq 20$ \\
\hline
\end{tabular}

\section{Screening experiment}

Of coarse and fine aggre gate, mineral powder, glass powder screening experiment. The file material gradation as shown in table 1-2

Table 1-2: Aggregate screening results table

\begin{tabular}{ccccccc}
\hline $\begin{array}{c}\text { Screen } \\
\text { size/mm }\end{array}$ & $\begin{array}{c}\mathrm{A}(10 \sim \\
15 \mathrm{~mm})\end{array}$ & $\begin{array}{c}\mathrm{B}(5 \sim \\
10 \mathrm{~mm})\end{array}$ & $\mathrm{C}(3 \sim 5 \mathrm{~mm}) \mathrm{D}(0 \sim 3 \mathrm{~mm})$ & filler & $\begin{array}{c}\text { glass } \\
\text { powder }\end{array}$ \\
\hline 16.00 & 99.04 & 100.00 & 100.00 & 100.00 & 100.00 & 100.00 \\
13.20 & 69.99 & 100.00 & 100.00 & 100.00 & 100.00 & 100.00 \\
9.50 & 9.13 & 99.61 & 100.00 & 100.00 & 100.00 & 100.00 \\
4.75 & - & 18.21 & 92.12 & 100.00 & 100.00 & 100.00 \\
2.36 & - & 1.61 & 16.06 & 81.72 & 100.00 & 100.00 \\
1.18 & - & 1.28 & 5.49 & 58.76 & 100.00 & 100.00 \\
0.60 & - & 1.14 & 3.55 & 37.65 & 100.00 & 100.00 \\
0.30 & - & 0.98 & 2.96 & 23.56 & 100.00 & 100.00 \\
0.15 & - & 0.85 & 2.61 & 15.88 & 99.40 & 95.23 \\
0.075 & - & 0.69 & 2.22 & 10.28 & 96.00 & 87.38 \\
\hline
\end{tabular}

\section{Gradation design}

After the completion of the screening of aggregate gradation design, mixture gradation design selection of AC-16I type, the composition design, two kinds of mixture set material mix ratio is A set of material, set $\mathrm{B}, \mathrm{C}$ aggregate, $\mathrm{D}$ and the ratio of the amount of mineral powder or glass powder is as follows: $34 \%, 16 \%, 10 \%, 36 \%, 4 \%$. The synthetic gradation curve and the design gradation range are shown in Figure 1-1, which is the same as the composition of the two kinds of mixture in Figure 1-1. 


\section{Figure 1-1 aggregate gradation design chart}

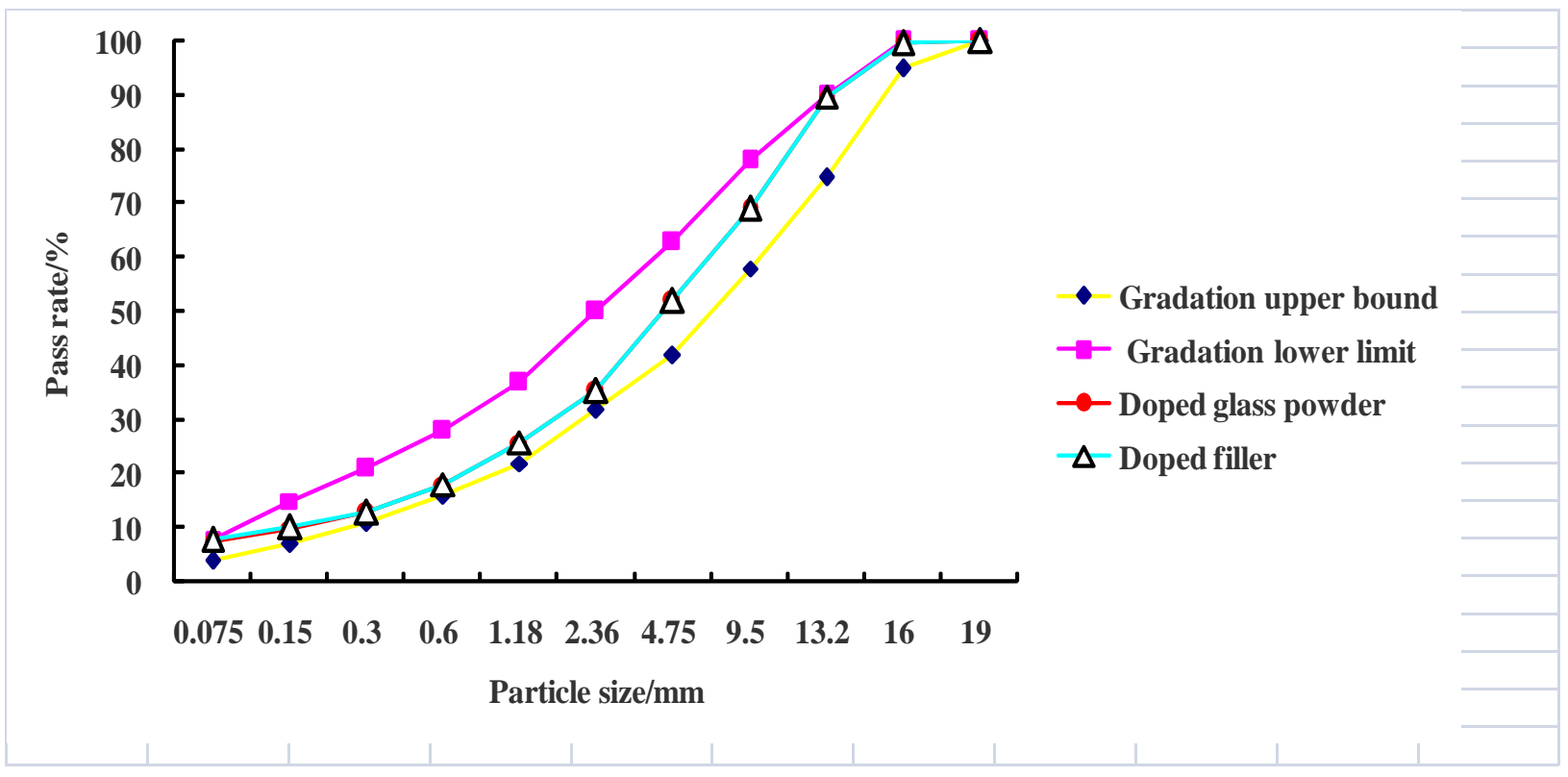

\section{Marshall experimental results analysis}

The mix proportion design of asphalt mixture has a great influence on the performance of asphalt concrete. According to the experimental standard, the mix proportion design of asphalt mixture and the mixture of asphalt mixture is designed. In this paper, the mix proportion design of asphalt mixture with the traditional and mixed with glass powder were $4.4 \%, 4.8 \%, 5.2 \%, 5.6 \%$, according to the experimental procedure of experiment. Through the experiment, mineral admixture and doped glass powder best asphalt amount in about $5 \%$.

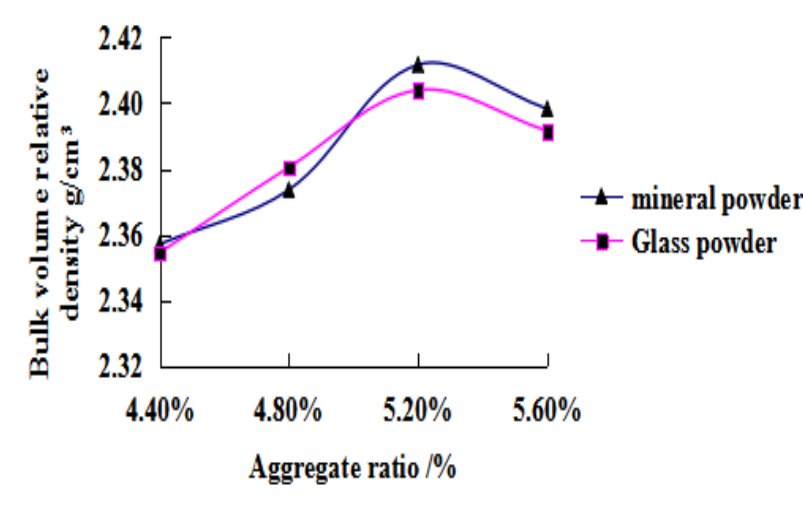

Figure 2-1: Bulk volume relative density change chart

From Figure 2-1 to figure 2-4, with the increase of asphalt content, the volume parameters of two kinds of asphalt mixture, which are traditional and mixed with glass powder, are similar and the variation trend is similar. In the process of asphalt dosage from $4.4 \%$ to two, the relative density and saturation of $5.2 \%$ kinds of asphalt mixture gradually increased and the maximum value was obtained at $5.2 \%$, then decreased gradually, and the porosity of two kinds of asphalt mixture gradually 
decreased and the minimum value was obtained. Glass powder instead of powder on the mixture volume parameters have little effect. It can be seen that the incorporation of glass powder will not affect the three-dimensional framework and the structure of the mixture, and will not cause the phenomenon of centrifugal or aggregate offset caused by mixing of different materials.

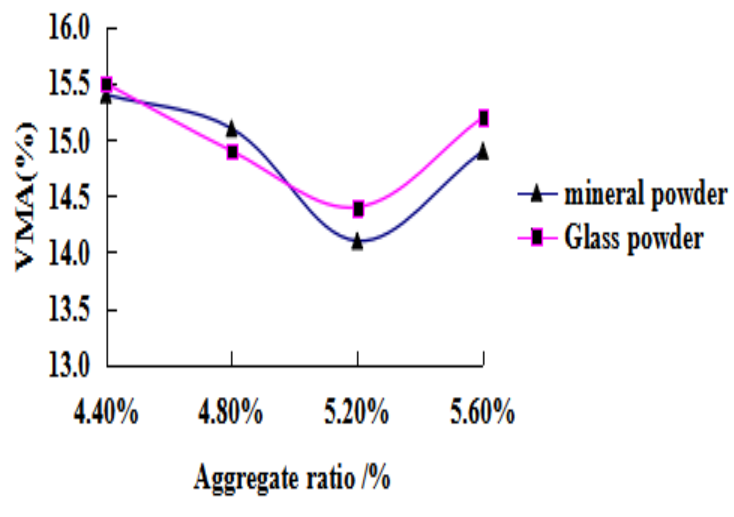

Figure 2-3: VMA change chart

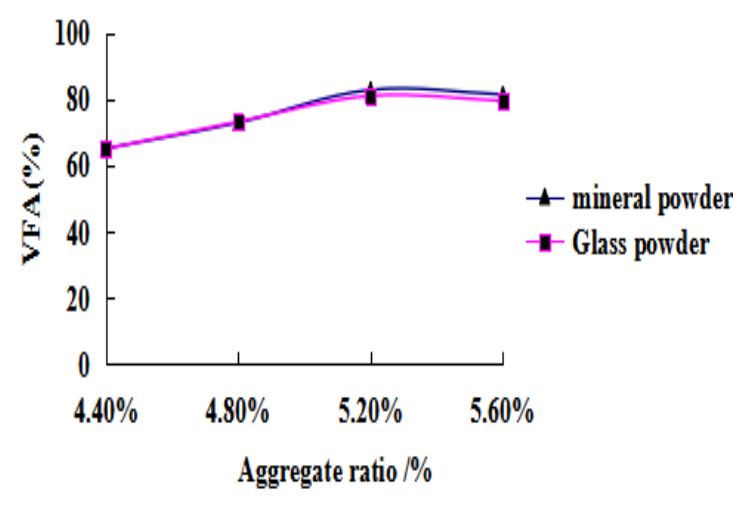

Figure 2-4: VFA change chart

\section{Marshall stability and flow value}

From Figure 2-5 and figure 2-6 shows, traditional asphalt mixture and glass doped powder asphalt mixture Marshall stability differ not quite, in asphalt content is about 5.0\% has the maximum value, and containing glass powder mixture instead flow value is larger, is conducive to the mixture mixing and paving. The reason is that the main ingredient of glass powder is not strong in the chemical adsorption and exchange between silica and asphalt, but the proportion of the structure of the glass powder and asphalt is small, and the proportion of free asphalt is larger.

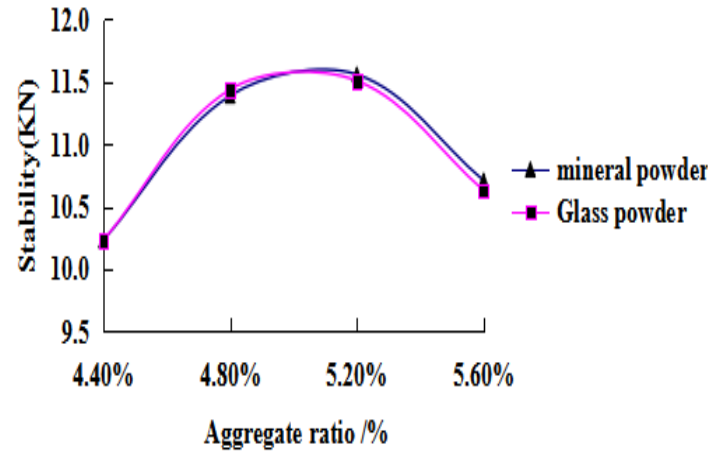

Figure2-5: Marshall stability change chart

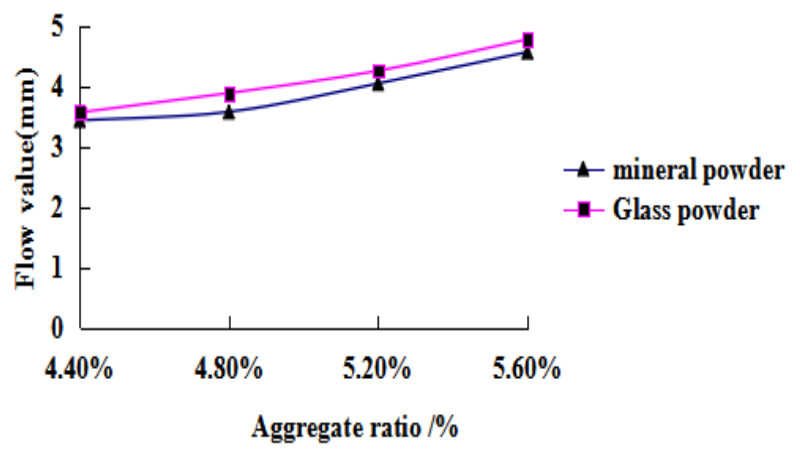

Figure 2-6: flow value change chart

\section{Wate r stability}

Marshall test of each mixture optimum asphalt aggregate ratio is determined, further the water stability of doped glass powder mixture experiment. The results were evaluated by water immersion Marshall test. Experimental results are as follows figure 3-1.

Doped glass powder asphalt mixture stability and slag asphalt mixture did not vary greatly, but doped glass powder asphalt mixture immersion stability is zero. Asphalt mixture of glass powder, the flow value of asphalt mixture ratio of powder flow value increased by $8.8 \%$.

Doped glass powder bituminous mixture test pieces after $48 \mathrm{~h}$ of immersion in the constant temperature of $60^{\circ} \mathrm{C}$ water bath, in front of the Marshall test load, the internal structure has been destroyed, basically have no bearing capacity.Experimental results are as follows figure 3-2. 

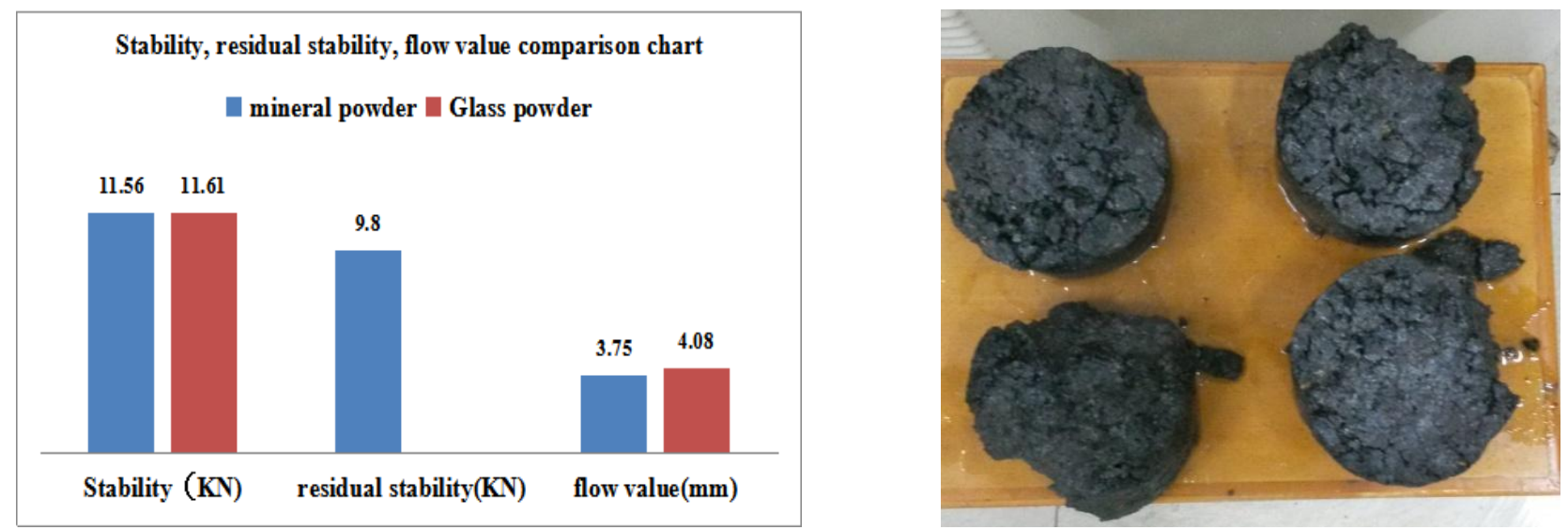

Figure 3-1: stability, residual stability, flow value comparison chart asphalt mixture is not loaded before the original graph

Figure 3-2: glas s powder

\section{The micro thermal method to explore the interaction of the aggregate and asphalt}

Peng Ying ${ }^{[10]}$ in the study of the interaction between asphalt and aggregate interface, the paper points out that the adsorption of the two kinds of media can be defined as the change in the concentration of the material composition in the phase interface and in its vicinity. This co ncentration change is caused by several different types of mutual attraction, which can be divided into mechanical, dynamic, static, thermal or chemical properties. The type of attraction determines the specific type of adsorption: chemical adsorption, electrostatic absorption, attachment, mechanical adsorption, etc..

The process of adsorption is exothermic. The size of the heat release depends on the nature of the interaction between the atoms and molecules and the solid surface. The size of the adsorption strength can be determined by measuring the quantity of heat released by the interaction between asphalt and filler particles.

The measured results are as follows: table 4-1

\begin{tabular}{cccccc}
\hline Filler type & $\begin{array}{c}\text { Proportion of } \\
\text { packing } \\
/ \mathrm{g} \cdot \mathrm{cm}^{-3}\end{array}$ & $\begin{array}{c}\text { Packing } \\
\text { sample } \\
\text { weight/g }\end{array}$ & $\begin{array}{c}\text { Maximum heat } \\
\text { release rate } \\
\text { calorie/g.s }) \\
\times 10^{-4}\end{array}$ & $\begin{array}{c}\text { Actual } \\
\text { reaction } \\
\text { time/s }\end{array}$ & $\begin{array}{c}\text { Total heat } \\
\text { release } \\
\text { (calorie/s }) \\
\times 10^{-2}\end{array}$ \\
\hline Glass powder & 2.30 & 1.00 & 1.4 & 725 & 1.5 \\
\hline Sandstone & 2.64 & 1.00 & 2.1 & 750 & 3.8 \\
\hline Dolomite & 2.83 & 1.00 & 2.2 & 750 & 3.7 \\
\hline Xuan Wuyan & 2.88 & 1.00 & 2.3 & 1100 & 4.8 \\
\hline Limestone & 2.88 & 1.00 & 2.8 & 1525 & 6.0 \\
\hline De lime & 2.30 & 0.50 & 16.7 & 3600 & 39.6 \\
\hline
\end{tabular}

As shown in table 4-1, the experimental results of trace heat release, the maximum heat release rate and time reaction time of limestone filler is 2 times that of glass powder, which is 4 times of the treated glass powder. The maximum heat release rate of the hydrated lime filler is about 6 times that of limestone, and the reaction time is about 2.5 times that of limestone, and about 5 times of the glass powder. The total amount of the released heat is 6.6 times that of limestone, and 26.4 times of glass powder. Thus experimental results showed that the glass powder and asphalt interface adsorption capacity very weak, with glass powder replace powder will seriously influence the asphalt mixture water stability, slaked lime to do for antistripping agent to improve the asphalt mixture water stability. 
Cause analysis: in accordance with mortar theory ${ }^{[7]}$, asphalt mixture is a dispersed system of three level space network structure. First of all, the coarse aggregate as the dispersed phase dispersed in asphalt mortar; secondly, asphalt mortar with fine aggregate for the dispersed phase dispersed in asphalt mortar; finally, asphalt and filler dispersed phase dispersed in the medium of asphalt. Three-level dispersed phase to asphalt mortar is the most important, the structure of mixture of the road performance plays a crucial role. And the AC type asphalt mixture is in accordance with continuous dense gradation design principle of typical suspended dense structure, this structure with larger particle size particles are smaller particles file a crowded open, not in direct contact with the formation of interlocking skeleton structure, separate suspended between the smaller particles and asphalt mastic and its strength is largely decided formed in the filler and asphalt asphalt mastic adhesive force ${ }^{[8-9]}$. Glass hydrophilic coefficient larger, in the water environment, water infiltration mixture, hydrophilic glass beads at the interface with the asphalt produced wide range of a thin film of water, and make the asphalt mortar discretization and the destruction of the binding force of glass powder and asphalt, makes AC type asphalt mixture the three-dimensional network structure of the basic level dispersed phase is destroyed and the whole mixture structure damage and the loss of capacity.

\section{Conclusions}

A .Doped glass powder and conventional asphalt mixture of optimum asphalt content did not appear to have a greater impact, that is, the change of ore type of asphalt mixture aggregate the optimum asphalt content without much impact.

B. Doped glass powder with traditional asphalt mixing material similar in different asphalt content of the volumetric parameters and the same change trend, 3D skeleton void structure, set segregation of asphalt migration direction similar.

C. Doped glass powder with traditional asphalt mixture stability similar, doped glass powder asphalt mixture flow value than asphalt mixture of the slag flow value increased by $8.8 \%$.

D. In water stability, doped glass powder asphalt mixture water stability range, before loading the internal structure has been damaged, glass powder to powder incorporated into asphalt mixture in must take corresponding anti stripping measures. Study on glass asphalt concrete suggestions of waste glass should be as fine aggre gate mixed in.

\section{Reference}

[1] Xu Meijun. Recycling and utilization of waste glass in the world: on the development of [J]. building materials, 2007 (1):51-55.

[2] Xu Meijun. Recycling and utilization of waste glass in the world: [J]. building materials development orientation, 2007 (2): 54-60.

[3] Day D E Sehaffer, R. Glassphalt Paving Handbook[M]. Jefferson:University of Missouri-Rolla , 1993

[4] Kanhdal P S Koehler, W S. The Ashalt Handbook[M]. [S.L.] : The Asphalt Institute Manual Series No4, 1989

[5] The Asphalt Handbook[J] The, Asphalt Institute Manual Series , 1989 (04): 10-12.

[6] Song Cheng, Zhang Jinxi, Wang Jian. Indoor experimental study on the performance of the asphalt concrete pavement with glass aggregate [J]. Journal of Wuhan University of Technology, 2011, 33 (12): 32-36.

[7] Shen Jinan. Performance of asphalt and asphalt mixture road [M]. Beijing: China Communications Press, 2001

[8] Deng Xuejun. [M]. Beijing: People's Communications Press.2008:336-337 
[9] Li Lihan. Beijing: People's communication press.2010:100+119,.

[10] Peng Ying. Study on [J]. technology of highway and transport of asphalt and aggre gate interface interaction, 2009 (2). 